05,12

\title{
Магнитные свойства и природа магнитного состояния интеркалированных соединений $\mathrm{Cr}_{x} \mathrm{MoSe}_{2}$
}

\author{
() В.Г. Плещев, Н.В. Селезнева
}

Институт естественных наук и математики Уральского фредерального университета им. Б.Н. Ельцина, Екатеринбург, Россия

E-mail: v.g.pleshchev@urfu.ru

(Поступила в Редакцию 9 октября 2018 г.)

Впервые синтезированы образцы диселенида молибдена, интеркалированного атомами хрома и исследованы их магнитные свойства в зависимости от концентрации интеркалянта, температуры и величины магнитного поля. Характер температурных зависимостей эффективных магнитных моментов и положительные значения парамагнитных температур Кюри в парамагнитной области указывают на возможные взаимодействия ферромагнитного типа между интеркалированными атомами.

Существование ферромагнитного состояния в соединениях $\mathrm{Cr}_{x} \mathrm{MoSe}_{2}$ при низких температурах подтверждено гистерезисными явлениями на температурных и полевых зависимостях намагниченности и магнитной восприимчивости.

Настоящая работа выполнена при финансовой поддержке Министерства образования и науки Российской Федерации (проект № 3.2916.2017/4.6) и РФФИ (проект № 16-02-00480).

DOI: 10.21883/FTT.2019.03.47238.274

\section{1. Введение}

В последнее время внимание исследователей направлено на создание и изучение материалов с пониженной размерностью, так как многие из них обладают уникальными характеристиками с точки зрения их возможного практического применения. К ним в частности относятся слоистые дихалькогениды переходных металлов (СДПМ) с общей формулой $M X_{2}$, где $M$ - переходный металл из 4,5 или 6 групп Периодической таблицы, $X$ - сера, селен или теллур [1].

К подобным системам могут быть отнесены и дихалькогениды молибдена $\mathrm{Mo}_{2}(X=\mathrm{S}, \mathrm{Se})$, имеющие квазидвумерный характер кристаллической структуры. Структурные различия, однако, состоят в том, что если для дихалькогенидов 4 группы характерна модификация $1 T$ с октаэдрической координацией атомов металла, то диселенид молибдена может иметь различные структурные модификации (политипы) такие как $2 \mathrm{H}-\mathrm{MoSe}_{2}$ и $3 R-\mathrm{MoSe}_{2}$, отличающиеся положением атомов и количеством слоев $\mathrm{MoSe}_{2}$ в элементарной ячейке. Модификация $2 \mathrm{H}-\mathrm{MoSe}_{2}$ описывается в рамках гексагональной структуры (пространственная группа $P 6_{3} / m m c$ ). Элементарная ячейка $2 \mathrm{H}-\mathrm{MoSe}_{2}$ содержит два слоя $\mathrm{MoSe}_{2}$ с тригонально-призматическим окружением атомов молибдена $[2,3]$.

По своим электрическим свойствам $\mathrm{MoSe}_{2}$ является полупроводником с шириной запрещенной зоны, изменяющейся в зависимости от количества слоев и составляющей около $1.5 \mathrm{eV}$ для монослоя и $1.1 \mathrm{eV}$ для объемного материала [4,5]. Слоистая структура таких материалов возникает вследствие различий в природе химической связи внутри трехслойных блоков $X-\mathrm{Mo}-X$ и между отдельными блоками, что определяет сильную анизотропию свойств вдоль и поперек слоев. Это облегчает процесс интеркаляции, который позволяет существенно модифицировать свойства подобных материалов. Ранее проводившиеся исследования показали, что $\mathrm{MoSe}_{2}$ может быть успешно интеркалирован щелочными, щелочноземельными и некоторыми переходными металлами [6,7]. При этом физические свойства соединений, получаемых путем интеркалирования, существенно отличаются от свойств исходных соединений $M X_{2}$ [8]. Характер изменения свойств интеркалированных соединений зависит как от сорта внедряемых $3 d$-атомов, так и от типа исходного соединения. Так при исследовании интеркалированных хромом диселенидов титана $\mathrm{Cr}_{x} \mathrm{TiSe}_{2}$ было показано, что образцы с содержанием хрома $x=0.5$ проявляли антиферромагнитное упорядочение ниже температуры Нееля $38 \mathrm{~K}$ [9], а в соединениях $\mathrm{Cr}_{0.5} \mathrm{TiTe}_{2}$ наблюдалось состояние ферромагнитного типа при температурах ниже $78 \mathrm{~K}$ [10]. В системе $\mathrm{Fe}_{x} \mathrm{TiSe}_{2}$ проявлялись антиферромагнитные свойства [11], а в $\mathrm{Fe}_{x} \mathrm{HfS}_{2}$ полевые зависимости намагниченности характеризовались гистерезисом, указывающим на наличие ферромагнитного состояния [12].

Магнитные свойства интеркалированных соединений на основе дихалькогенидов молибдена изучены очень слабо. Имеются данные о синтезе и свойствах соединений на основе дисульфида молибдена, интеркалированного переходными $3 d$-элементами $(\mathrm{Ni}, \mathrm{V}, \mathrm{Cr}, \mathrm{Fe}, \mathrm{Co})$ при их концентрации, соответствующей общей формуле $M(3 d) \mathrm{Mo}_{2} \mathrm{~S}_{4}[7,13,14]$, где в $\mathrm{FeMo}_{2} \mathrm{~S}_{4}$ в результате магнитных и нейтронографических исследований было установлено антиферомагнитное состояние при температурах ниже $150 \mathrm{~K}$.

Эти данные показывают, что дальнейшие исследования дихалькогенидов молибдена, интеркалированных $3 d$-переходными металлами, представляют несомненный 
Таблица 1. Значения параметров элементарной ячейки $a$ и $c$ соединений $\mathrm{Cr}_{x} \mathrm{MoSe}_{2}$

\begin{tabular}{l|l|l}
\hline$x$ & $a, \AA$ & $c, \AA$ \\
\hline 0 & 3.289 & 12.927 \\
0.1 & 3.287 & 12.926 \\
0.20 & 3.287 & 12.922 \\
0.25 & 3.286 & 12.920 \\
0.5 & 3.284 & 12.909
\end{tabular}

интерес. Настоящая работа посвящена изучению природы магнитного состояния соединений $\mathrm{Cr}_{x} \mathrm{MoSe}_{2}$ в широкой области концентраций.

\section{2. Эксперимент}

Образцы интеркалированных соединений $\mathrm{Cr}_{x} \mathrm{MoSe}_{2}$ синтезировались методом твердофазных реакций в вакуумированных кварцевых ампулах. Приготовление образцов включало две стадии: на первой из отдельных элементов (молибдена марки М0 и селена марки ОСЧ) синтезировалось соединение $\mathrm{MoSe}_{2}$, которое служило матрицей для приготовления интеркалированных соединений, на второй - осуществлялся синтез интеркалированных фаз путем твердофазного синтеза $\mathrm{MoSe}_{2}$ с необходимым количеством металлического хрома, полученного электролитическим методом. Температура, при которой осуществлялся синтез интеркалированных фаз, составляла $1100 \mathrm{~K}$. Процедура получения готовых продуктов, помимо первичных отжигов, включала серию гомогенизационных отжигов образовавшихся продуктов. Аттестация образцов осуществлялась рентгенографически на дифрактометре Bruker D8 Advance в $\mathrm{Cu} K_{\alpha}$ излучении. Обработка рентгенографических данных производилась с использованием программного пакета GSAS. Как показали расчеты, кристаллическая структура полученных образцов в основном соответствует структуре $2 \mathrm{H}-\mathrm{MoSe}_{2}$. Значения параметров элементарной ячейки для образцов различного состава приведены в табл. 1. Погрешности в определении параметров составили $\Delta a= \pm 0.003 \AA$ и $\Delta c= \pm 0.005 \AA$. Значения параметров для синтезированной матрицы $\mathrm{MoSe}_{2}$ находятся в интервале имеющихся данных, полученных в различных работах $[2,15,16]$. Как следует из приведенных данных, при возрастании содержания хрома изменение параметра $a$ невелико и находится в пределах экспериментальной погрешности. Изменение параметра с более значительно, а его уменьшение является следствием возникающего взаимодействия $3 d$-орбиталей хрома со слоями $\mathrm{MoSe}_{2}$. Подобное сжатие элементарной ячейки наблюдалось ранее и для других соединений, интеркалированных $3 d$-переходными элементами.

Измерения магнитных свойств образцов осуществлялись с помощью СКВИД-магнетометра фирмы Quantum Design (MPMS) в интервале температур 2-350 K и в магнитных полях до $70 \mathrm{kOe}$ Температурные измерения магнитной восприимчивости производились в магнитном поле $1 \mathrm{kOe}$ при охлаждении без магнитного поля (режим $Z F C$ ) и при охлаждении в магнитном поле (режим $F C$ ).

\section{3. Результаты}

Измерения температурных зависимостей намагниченности и магнитной восприимчивости при использовании режимов $Z F C$ и $F C$ показали, что в области температур выше $100 \mathrm{~K}$ зависимости при нагревании и охлаждении являются полностью обратимыми и их вид представлен на рис. 1. В то же время зависимости обратной восприимчивости от температуры в той же температурной области обнаруживают отклонения от предполагавшейся линейной зависимости при температурах ниже $150 \mathrm{~K}$, как это показано на рис. 2. Причиной подобных отклонений может быть наличие взаимодействия между ионами

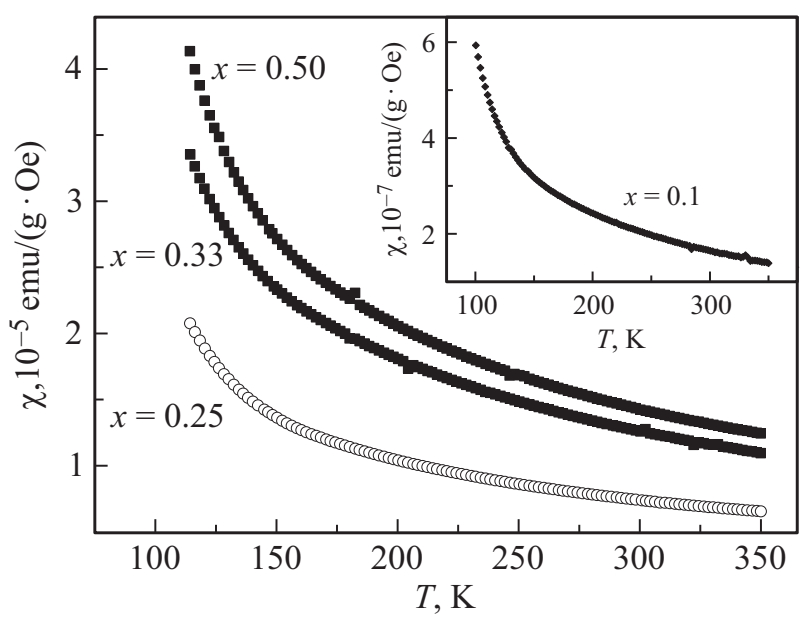

Рис. 1. Температурные зависимости магнитной восприимчивости соединений $\mathrm{Cr}_{x} \mathrm{MoSe}_{2}$ в высокотемпературной области.

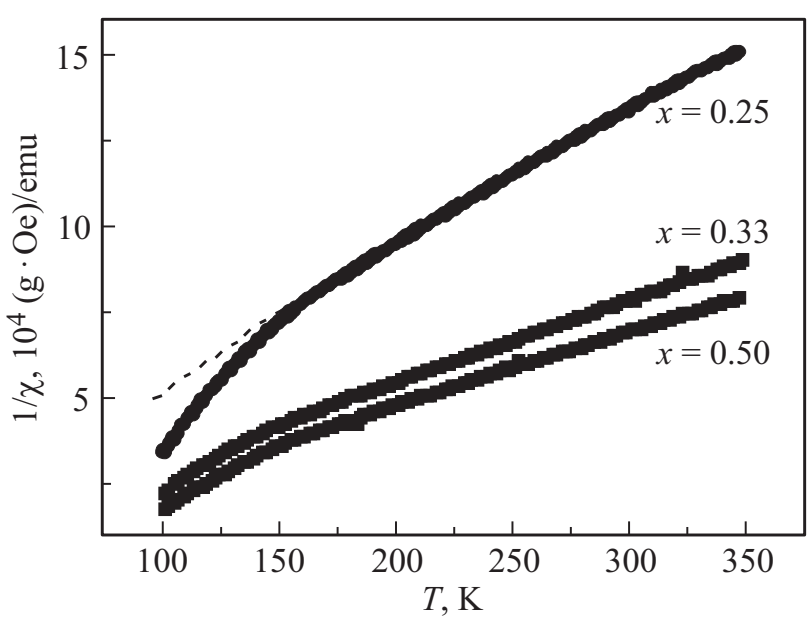

Рис. 2. Температурные зависимости обратной магнитной восприимчивости соединений $\mathrm{Cr}_{x} \mathrm{MoSe}_{2}$ в высокотемпературной области. 
Таблица 2. Значения температурно-независимого вклада в магнитную восприимчивость $\chi_{0}$, парамагнитной температуры Кюри $\Theta_{p}$, эффективного магнитного момента $\mu_{\text {eff }}$ на ион хрома образцов $\mathrm{Cr}_{x} \mathrm{MoSe}_{2}$

\begin{tabular}{l|c|c|c}
\hline \multicolumn{1}{c|}{$x$} & $\chi_{0}, 10^{-6} \mathrm{emu} /(\mathrm{g} \cdot \mathrm{Oe})$ & $\Theta_{p}, \mathrm{~K}$ & $\mu_{\mathrm{eff}}, \mu_{\mathrm{B}}$ \\
\hline 0.1 & 0.06 & 10 & 0.95 \\
0.2 & 0.7 & 8 & 3.52 \\
0.25 & 3.2 & 15 & 3.75 \\
0.33 & 5.5 & 50 & 4.05 \\
0.5 & 6.8 & 43 & 3.93
\end{tabular}

хрома, как это ранее сообщалось для других подобных систем на основе дихалькогенидов титана $[17,18]$.

Обработка экспериментальных зависимостей $\chi(T)$ в данной области температур производилась в соответствии с выражением для обобщенного закона Кюри-Вейсса

$$
\chi(T)=\chi_{0}+C /\left(T-\Theta_{p}\right),
$$

где $\chi_{0}=\chi_{p}+\chi_{d}-$ член, обусловленный паулиевским парамагнетизмом электронов проводимости $\left(\chi_{p}\right)$ и диамагнитным вкладом $\left(\chi_{d}\right) ; C-$ постоянная Кюри; $\Theta_{p}-$ парамагнитная температура Кюри, знак и величина которой отражают среднее значение алгебраической суммы обменных взаимодействий разного знака между локализованными моментами. В данной аппроксимации величина $\chi_{0}$ считалась независящей от температуры. Значения параметров в уравнении (1), а также значения эффективных магнитных моментов на ион хрома показаны в табл. 2. Результаты расчетов показали, что значения $\chi_{0}$ возрастают по мере увеличения содержания хрома в соединениях. Поскольку изменение диамагнитного вклада, происходящего за счет увеличения содержания атомов хрома, можно считать незначительным, то увеличение $\chi_{0}$ главным образом связано с паулиевским вкладом в восприимчивость и может указывать на возрастание плотности состояний на уровне Ферми. Эффективные магнитные моменты увеличиваются при росте содержания хрома, приближаясь к значению, соответствующему спиновому вкладу для иона $\mathrm{Cr}^{3+}$. Парамагнитная температура Кюри также возрастает по величине при увеличении концентрации хрома и имеет положительный знак для всех образцов, что отражает преобладающий вклад взаимодействий ферромагнитного типа. Дополнительное подтверждение возможности такого взаимодействия было нами получено из характера температурных зависимостей эффективных магнитных моментов в интервале $100-350 \mathrm{~K}$, которые были рассчитаны по формуле

$$
\mu_{\mathrm{eff}}=\left(8 \chi_{\mathrm{mol}} T / x\right)^{1 / 2},
$$

где $\chi_{\text {mol }}-$ молярная восприимчивость соединений, $T-$ температура, $x$ - содержание хрома в $\mathrm{Cr}_{x} \mathrm{MoSe}_{2}$. В этом выражении значения $\chi_{\text {mol }}$ были получены из удельной величины $\chi$ за вычетом температурно независимой составляющей $\chi_{0}$. Это позволило при расчете $\mu_{\text {eff }}$ учесть лишь кюри-вейссовский вклад (второе слагаемое в выражении 1). Результаты расчетов для образцов различного состава представлены на рис. 3. Видно, что в области температур, превышающих $150 \mathrm{~K}$, значения $\mu_{\text {eff }}$ остаются постоянными и практически совпадают по величине с теми, которые представлены в табл. 2. Из этого можно заключить, что эта область температур соответствует парамагнитному состоянию образцов. По мере уменьше-

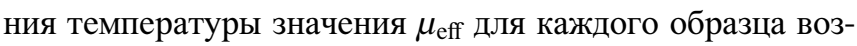
растают, что характерно для систем, обнаруживающих при понижении температуры обменное усиление парамагнетизма вследствие возникновения ферромагнитного характера взаимодействия между магнитоактивными ионами. При более низких температурах зависимости $\chi(T)$ полученные при измерениях в режимах $Z F C$ и $F C$ у всех образцов оказываются необратимыми (рис. 4). Зависимости, полученные в режиме $Z F C$, обнаруживают

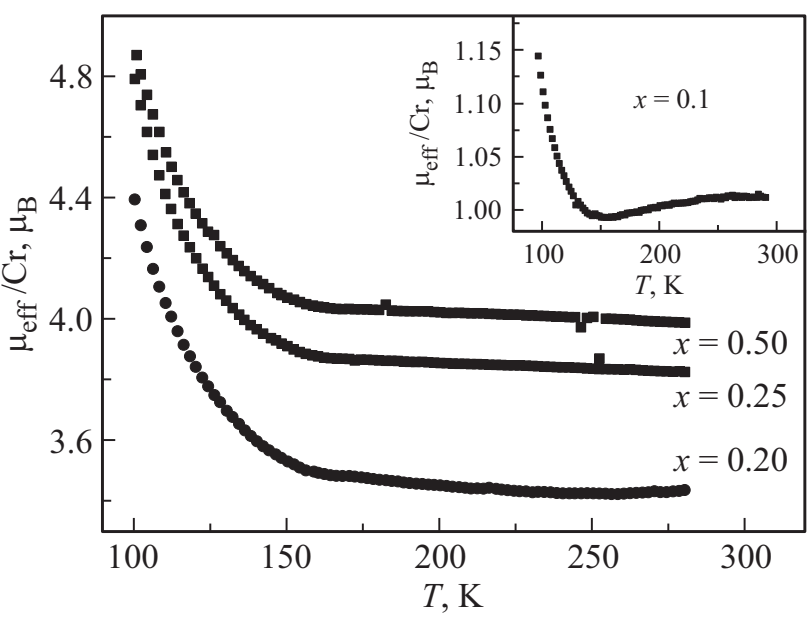

Рис. 3. Температурные зависимости эффективных магнитных моментов на ион хрома в соединениях $\mathrm{Cr}_{x} \mathrm{MoSe}_{2}$.

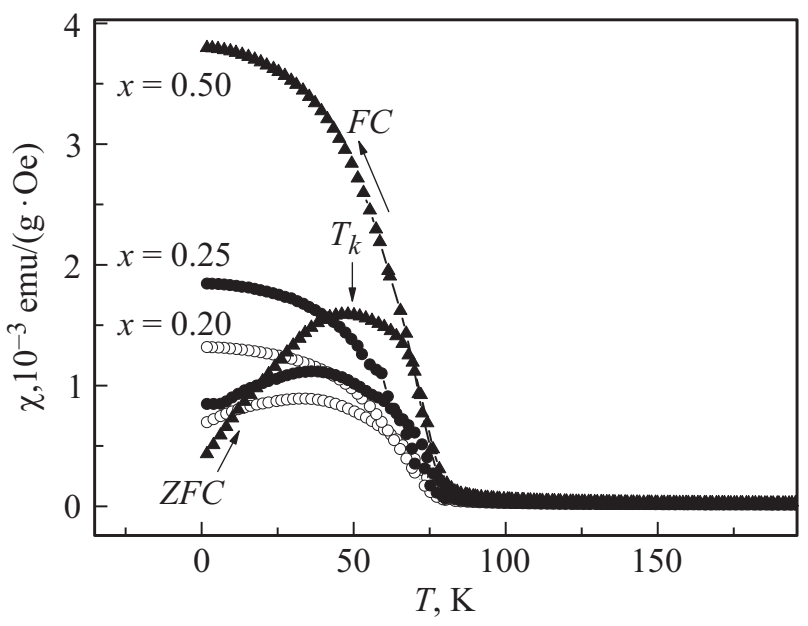

Рис. 4. Температурные зависимости магнитной восприимчивости соединений $\mathrm{Cr}_{x} \mathrm{MoSe}_{2}$, измеренные в магнитном поле $1 \mathrm{kOe}$ в режимах $Z F C$ и $F C$. 
Таблица 3. Значения температур магнитных превращений $T_{k}$, удельных магнитных моментов, интерполированных к нулевому магнитному полю $m_{0}$, магнитной восприимчивости парапрцесса $\chi_{p p}$ и локализованных магнитных моментов на ион хрома $\mu_{s}$ образцов $\mathrm{Cr}_{x} \mathrm{MoSe}_{2}$

\begin{tabular}{l|c|c|c|c}
\hline \multicolumn{1}{c|}{$x$} & $T_{k}, \mathrm{~K}$ & $m_{0}, \mathrm{emu} / \mathrm{g}$ & $\chi_{p p}, 10^{-6} \mathrm{emu} /(\mathrm{g} \cdot \mathrm{Oe})$ & $\mu_{s}, \mu_{\mathrm{B}}$ \\
\hline 0.1 & 28 & 0.537 & 7.2 & 0.25 \\
0.2 & 34 & 2.17 & 21.7 & 0.35 \\
0.25 & 35 & 3.02 & 24.6 & 0.51 \\
0.33 & 38 & 2.83 & 40.2 & 0.42 \\
0.5 & 50 & 6.25 & 53.2 & 0.64
\end{tabular}

широкий максимум при температурах $T_{k}$, положение которого смещается в область более высоких температур при росте содержания хрома (табл. 3). Полученные данные могут свидетельствуют о том, что в соединениях $\mathrm{Cr}_{x} \mathrm{MoSe}_{2}$ при охлаждении ниже данных температур возникает состояние типа кластерного спинового стекла с преимущественно ферромагнитной связью между отдельными магнитными моментами. В этом случае температуры $T_{k}$ могут соответствовать температурам замерзания спинового стекла. Однако при переходе к спин-стекольному состоянию этот максимум должен быть более острым, как это было обнаружено для диселенида титана, интеркалированного атомами хрома $[9,19]$. Кроме того, формирование спинстекольного состояния, как правило, должно наблюдаться при малой концентрации магнитоактивных атомов в некоторой немагнитной матрице. Видно также, что при охлаждении образцов в сравнительно небольшом магнитном поле $1 \mathrm{kOe}$ (режим $F C$ ) магнитная восприимчивость возрастает монотонно до $T=2 \mathrm{~K}$, не проявляя никаких особенностей, связанных с обратным переходом в спинстекольное состояние. Можно также отметить, что соотношение между величинами восприимчивости при $T=2 \mathrm{~K}$, полученных в режимах ZFC и $F C\left(\chi_{\mathrm{FC}} / \chi_{\mathrm{ZFC}}\right)$, возрастает при увеличении содержания хрома в образцах и достигает десяти раз для $\mathrm{Cr}_{0.5} \mathrm{MoSe}_{2}$. Все эти факты требуют иного подхода к объяснению наблюдаемых особенностей. Полевые зависимости магнитных свойств могут дать дополнительную информацию о природе магнитного состояния соединений $\mathrm{Cr}_{x} \mathrm{MoSe}_{2}$.

Кривые намагничивания образцов $\mathrm{Cr}_{x} \mathrm{MoSe}_{2}$, измеренные при $T=2 \mathrm{~K}$, приведенные на рис. 5, характеризуются тем, что у всех образцов намагниченность $(M)$, увеличивающаяся с ростом содержания хрома, не достигает насыщения даже при увеличении магнитного поля до $70 \mathrm{kOe}$, что указывают на значительный парапроцесс. В этом случае рост величины $M$ происходит за счет роста истинной намагниченности, характерного для ферромагнитного состояния. В области магнитных полей превышающих $30 \mathrm{kOe}$ зависимости $M(H)$ имеют линейный характер, и могут быть выражены как $M=m_{0}+\chi_{p p} H$, где $m_{0}$ характеризует удельный магнитный момент образца, аппроксимированный к нулевому магнитному полю, а коэффициент $\chi_{p p}$ соответствует магнитной восприимчивости парапроцесса. Эти данные позволили определить величины спонтанного магнитного момента $\mu_{s}$, приходящегося на ион хрома в каждом соединении. Результаты аппроксимации и расчетов представлены в табл. 3.

При дифференцировании начального участка кривых намагничивания можно было определить величины поля, в котором магнитная восприимчивость достигает максимальной величины. Характерные зависимости $d M / d H$ (на вставке рис. 5) показывают, что $\chi_{\max }$ достигается в полях около 1000 Ое, которые сравнимы с величиной магнитного поля, использованного при измерениях зависимостей $\chi(T)$ (рис. 4). Основываясь на этих данных, можно полагать, что необратимые изменения, показанные на рис. 4, скорее всего, связаны с проявлением температурного магнитного гистерезиса, характерного для ферромагнитных материалов. Природа этого явления определяется температурной зависимостью магнитной анизотропии. Учитывая анизотропный характер кристаллической структуры интеркалированных соединений, это объяснение представляется вполне обоснованным. Ферромагнитное состояние при низких температурах подтверждается наличием петель магнитного гистерезиса при знакопеременном изменении магнитного поля (рис. 6). Эти петли для всех образцов имеют симметричный вид и характеризуются коэрцитивной силой около $3 \mathrm{kOe}$. Площадь петель гистерезиса при увеличении содержания хрома возрастает главным образом за счет увеличения намагниченности.

Следует отметить существенные различия между значениями эффективных магнитных моментов $\mu_{\text {eff }}$ на ионе хрома, определенных в области парамагнитного состояния и локализованных моментов $\mu_{s}$, полученных при экстраполяции кривых намагничивания к нулевому полю. Различие между этими величинами, хотя и в меньшей степени, отмечалось ранее для $\mathrm{Cr}_{x} \mathrm{TiSe}_{2}$ [4]. Это может быть связано со значительной делокализацией

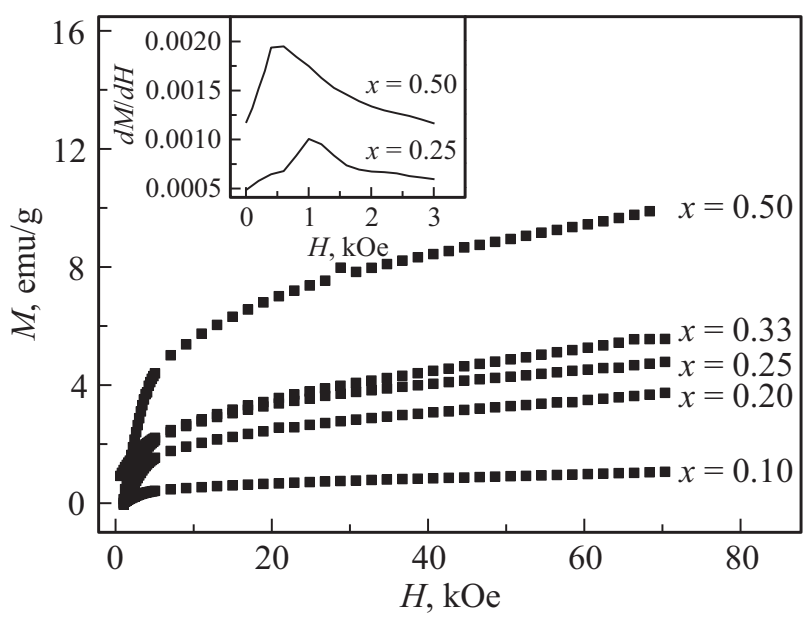

Рис. 5. Кривые намагниченности соединений $\mathrm{Cr}_{x} \mathrm{MoSe}_{2}$ при $T=2 \mathrm{~K}$. На вставке: зависимости производной $d M / d H$ от величины магнитного поля. 


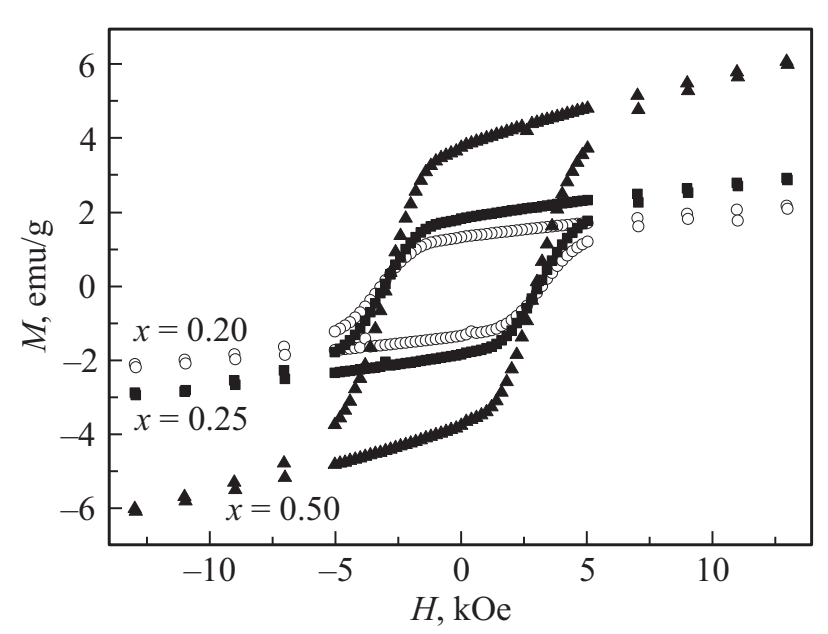

Рис. 6. Петли магнитного гистерезиса соединений $\mathrm{Cr}_{x} \mathrm{MoSe}_{2}$ при $T=2 \mathrm{~K}$.

$3 d$-электронов хрома. В связи с этим для адекватного описания магнитного состояния исследуемых соединений помимо модели локализованных моментов возможно следует привлекать представления о флуктуациях спиновой плотности в рамках зонной модели [20,21]. Подтверждением этому может служить отмеченное выше увеличение значений $\chi_{0}$, Кроме того, в результате контрольных измерений электросопротивления образцов было обнаружено уменьшение удельного электросопротивления и переход от полупроводникового поведения к металлическому типу при увеличении содержания хрома в образцах.

\section{4. Заключение}

Впервые проведенные систематические исследования магнитных свойств интеркалированных соединений $\mathrm{Cr}_{x} \mathrm{MoSe}_{2}(x=0.1-0.5)$ позволили получить новые данные относительно магнитного состояния этих материалов. Было определено, что парамагнитное состояние образцов существует в области температур превышающих $150 \mathrm{~K}$. При аппроксимации температурных зависимостей магнитной восприимчивости в высокотемпературной области в соответствии с законом Кюри-Вейсса, было определено, что парамагнитные температуры Кюри всех исследованных соединений имели положительный знак, характеризуя возможность взаимодействий ферромагнитного типа между магнитоактивными ионами. Анализ температурных зависимостей эффективных магнитных моментов в области $100-350 \mathrm{~K}$ для различных образцов дал дополнительные свидетельства о проявлении такого взаимодействия в виде обменного усиления парамагнетизма при понижении температуры. Значения

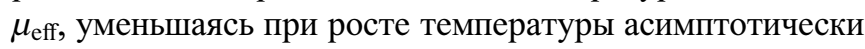
приближаются к постоянным значениям при температурах, превышающих $150 \mathrm{~K}$, свидетельствуя о переходе образцов к парамагнитному состоянию.
В результате низкотемпературных исследований было подтверждено формирование магнитного упорядочения. На температурных зависимостях магнитной восприимчивости в низкотемпературной области обнаружены гистерезисные явления при измерении в режимах $Z F C$ и $F C$, являющиеся проявлением температурного магнитного гистерезиса. При этом значения температур $T_{k}$, соответствующих максимумам на зависимостях $\chi(T)$ в режиме $Z F C$ и характеризующих изменение магнитного состояния, возрастают при увеличении содержания хрома в образцах.

Кривые намагничивания образцов измеренные при $T=2 \mathrm{~K}$ в магнитных полях до $70 \mathrm{kOe}$ демонстрируют отсутствие насыщения и обнаруживают довольно сильный парапроцесс с линейной зависимостью $M(T)$ в области больших полей. При аппроксимация этих зависимостей к нулевому полю были определены значения локализованных магнитных моментов на ионах хрома $\left(\mu_{s}\right)$ в исследованных соединениях, а также величины магнитной восприимчивости парапроцесса $\left(\chi_{p p}\right)$, ворастающие при увеличении содержания хрома в образцах. Петли магнитного гистерезиса, измеренные при $T=2 \mathrm{~K}$, имеют вид, характерный для ферромагнитного состояния с величиной коэрцитивной силы около $3 \mathrm{kOe}$. Таким образом в результате проведенных исследований установлено, что в области температур 150-350 K интеркалированные соединения $\mathrm{Cr}_{x} \mathrm{MoSe}_{2}$ находятся в парамагнитном состоянии, а при более низких температурах происходит возрастание обменных взаимодействий, приводящих к формированию магнитного порядка ферромагнитного типа с характерными чертами магнитного и термомагнитного гистерезиса.

Существенно меньшие величины локализованных магнитных моментов $\mu_{s}$ по сравнению с эффективными магнитных моментов $\mu_{\text {eff }}$, полученных в области парамагнитного состояния соединений $\mathrm{Cr}_{x} \mathrm{MoSe}_{2}$ вероятно свидетельствует о том, что для адекватного описания магнитного состояния помимо модели локализованных моментов возможно следует привлекать представления зонной модели. Кроме того, для установления особенностей магнитного порядка в этих соединениях необходимо проведение детальных нейтронографических исследованияй.

\section{Список литературы}

[1] Л.А. Чернозатонский, А.А. Артюх. УФН 188, 3 (2018).

[2] P.B. James, M.T. Lavik. Acta Cryst. 16, 1183 (1963).

[3] В.Л. Калихман, Я.С. Уманский. УФН 108, 503 (1972).

[4] Th. Boker, R. Severin. Phys. Rev. B 64, 235305 (2001).

[5] H. Lee, Ji.H. Kim, Ch.J. Lee. Appl. Phys. Lett. 109, 222105 (2016).

[6] J. Morales, J. Santos, K.L. Tirado. Solid State Ionics 83, 57 (1996).

[7] P.A. Berseth, Th.A. Hughes, R. Schneidmiller, A Smalley, D.C. Johnson. Solid State Sci. 4, 717 (2002).

[8] M. Inoue, H.P. Hughes, A.D. Yoffe. Adv. Phys. 38, 565 (1989).

[9] V.G. Pleschov, N.V. Baranov, A.N. Titov, K. Inoue, M.I. Bartashevich, T. Goto. J. Alloys Comp. 320, 13 (2001). 
[10] В.Г. Плещев, А.В. Королев, Ю.А. Дорофеев. ФТТ 46, 282 (2004).

[11] Н.В. Селезнева, Н.В. Баранов, В.Г. Плещев, Н.В. Мушников, В.И. Максимов. ФТТ 53, 269 (2011).

[12] В.Г. Плещев, Н.В. Селезнева. ФТТ 60, 245 (2018).

[13] P. Vaquero, M.L. Kosidowski, A.V. Powell. Chem. Mater. 14, 1201 (2002).

[14] Par C. d'Anterroches Meneau, B. Boucher. Acta Cryst. B 33, 1495 (1977).

[15] S.K. Srivastava, B.N. Avasthi. J. Mater. Sci. 24, 1919 (1989).

[16] M.M. Vora, A.M. Vora. J. Semicond. 33, 012001-5 (2012).

[17] Н.В. Баранов, В.Г. Плещев, Е.М. Шерокалова, Н.В. Селезнева, А.С. Волегов. ФТТ 53, 654 (2011).

[18] Н.В. Баранов, В.Г. Плещев, А.Н. Титов, В.И. Максимов, Н.В. Селезнева, Е.М. Шерокалова. Нанотехника 3, 15 (2008).

[19] В.Г. Плещев, Н.В. Селезнева, В.И. Максимов, А.В. Королев, А. Подлесняк, Н.В. Баранов. ФТТ 51, 885 (2009).

[20] T. Moriya. J. Magn. Magn. Mater. 14, 1 (1979).

[21] Р.З. Левитин, А.С. Маркосян. УФН 155, 623 (1988).

Редактор Т.Н. Василевская 